PROCEEDINGS OF THE

AMERICAN MATHEMATICAL SOCIETY

Volume 139, Number 5, May 2011, Pages 1787-1803

S 0002-9939(2010)10812-2

Article electronically published on December 13, 2010

\title{
THE RUNGE THEOREM FOR SLICE HYPERHOLOMORPHIC FUNCTIONS
}

\author{
FABRIZIO COLOMBO, IRENE SABADINI, AND DANIELE C. STRUPPA
}

(Communicated by Franc Forstneric)

\begin{abstract}
In this paper we introduce and study rational slice monogenic functions. After proving a decomposition theorem for such functions, we are able to prove the Runge approximation theorem for slice monogenic functions. We then show how a similar argument can be used to obtain an analogue of the Runge approximation theorem in the slice regular setting.
\end{abstract}

\section{INTRODUCTION}

Slice hyperholomorphic functions, i.e. slice regular and slice monogenic functions, were introduced in [8, [16, 17] and further studied in a series of papers; see e.g. 2], 9, 10, 11, [13. In their recent work [18, the authors show that an alternative definition which works for functions with values in any real alternative algebra can be given in terms of what they call (following the terminology introduced by Cullen in [14) stem functions. To be precise, the idea of stem functions is already present in the early works of Fueter (see [15]) which eventually led to his theory of Fueter regular functions. This theory is now very well understood, and the properties of Fueter regular functions in one or several variables are studied e.g. in [6]. As it turns out the slice regular functions of [18] are a slightly more restrictive class than the one of [16, but more natural and thus more suitable to their analysis. In this paper we will always consider slice hyperholomorphic functions, i.e. slice monogenic or slice regular, in the sense of [18].

Specifically we show that slice hyperholomorphic functions can be approximated by polynomials (in the spirit of the classic Runge theorem) on suitable open sets in $\mathbb{R}^{n+1}$. Unlike what happens with the standard hyperholomorphic functions (either monogenic or regular), however, not every open set is a domain of hyperholomorphy. In fact, we know that the natural domains for slice hyperholomorphic functions are the axially symmetric domains. We will show that any axially symmetric domain is a domain of regularity.

We first study the case of slice monogenic functions. Since a Clifford algebra $\mathbb{R}_{n}$ contains zero divisors when $n \geq 3$, we will need more restrictive hypotheses in order to define rational functions, while in the case of the algebra of quaternions,

Received by the editors May 25, 2010.

2010 Mathematics Subject Classification. Primary 30G35; Secondary 30B10, 30C10.

(C) 2010 American Mathematical Society
Reverts to public domain 28 years from publication 1787 
rational functions can be defined under weaker assumptions. Thus we prove the approximation theorems in the case of slice monogenic functions and we then show, in the last section, how to modify the statements in order to obtain the results in the case of slice regular functions.

We conclude this introduction by pointing out that slice regular and slice monogenic functions are interesting not only for their intrinsic value, but more importantly because they are the natural tool to define a functional calculus for noncommuting operators, 7], as well as a functional calculus for quaternionic operators; see [3, 4], 5].

\section{Slice monogenic FunCtions}

The purpose of this section is to provide basic notions about slice monogenic functions. By $\mathbb{C}$ we mean the complex plane whose variable is denoted by $z=u+i v$, and by $\mathbb{R}_{n}$ we denote the Clifford algebra over $n$ imaginary unit $e_{1}, \ldots, e_{n}$ such that $e_{i} e_{j}+e_{j} e_{i}=-2 \delta_{i j}$, where $\delta_{i j}$ denotes the Kronecker's delta. As is customary, we will denote by $e_{A}=e_{i_{1}} \ldots e_{i_{r}}$ a generator of $\mathbb{R}_{n}$ as a real vector space. The index $A$ denotes the ordered set $\left\{i_{1}, \ldots, i_{r}\right\}$ in the power set $\wp(1, \ldots, n)$ where $i_{1}<\ldots<i_{r}$, while for $A=\emptyset$ we set $e_{\emptyset}=1$. An element in $\mathbb{R}_{n}$ is written as $a=\sum_{A} a_{A} e_{A}$, $a_{A} \in \mathbb{R}$, and it is obvious that the dimension of the real vector space $\mathbb{R}_{n}$ is $2^{n}$. The elements of the form $\sum_{i=1}^{n} a_{i} e_{i}$ are called 1-vectors or vectors and can be identified with $\left(a_{1}, \ldots, a_{n}\right) \in \mathbb{R}^{n}$, while the elements of the form $a_{0}+\sum_{i=1}^{n} a_{i} e_{i}$ are called paravectors and can be identified with $\left(a_{0}, \ldots, a_{n}\right) \in \mathbb{R}^{n+1}$. An element $a=\sum_{A} a_{A} e_{A}$, where the length $|A|$ of the index $A$ is $k$, is called a $k$-vector. Any Clifford number can be written by putting in evidence its $k$-vector part, denoted by $[\cdot]_{k}, k=0, \ldots, n$, as $a=[a]_{0}+[a]_{1}+\ldots+[a]_{n}$; note that $[a]_{0}$ corresponds to the scalar part of $a$. The conjugate $a^{c}$ of an element $a \in \mathbb{R}_{n}$ is defined by $e_{i}^{c}=-e_{i}$, $\left(e_{i} e_{j}\right)^{c}=e_{j} e_{i}$ and then by natural extension. We obtain that

$$
a^{c}=[a]_{0}-[a]_{1}-[a]_{2}+[a]_{3}+\ldots,
$$

where the signs are repeated with periodicity four. The vectors of norm 1 form an $(n-1)$-sphere defined by

$$
\mathbb{S}=\mathbb{S}_{n-1}=\left\{a_{1} e_{1}+\ldots+a_{n} e_{n} \mid \sum_{i=1}^{n} a_{i}^{2}=1\right\} .
$$

For any choice of an element $I \in \mathbb{S}$ the set of paravectors of the form $u+I v$, $u, v \in \mathbb{R}$, spans a complex plane $\mathbb{C}_{I}$. In particular, the notation $\mathbb{C}_{I}^{+}$will denote the half plane defined by

$$
\mathbb{C}_{I}^{+}=\{u+I v \mid u, v \in \mathbb{R}, v \geq 0\} .
$$

Among the open sets in $\mathbb{R}^{n+1}$ there are some that are important in our theory since they are the natural domains on which slice regular functions will be defined.

Definition 2.1. Let $\Omega \subseteq \mathbb{R}^{n+1}$. We say that $\Omega$ is axially symmetric if, for all $u+I v \in \Omega$, the $(n-1)$-sphere $u+\mathbb{S} v=\{u+J v \mid J \in \mathbb{S}\}$ is contained in $\Omega$.

Given an open set $D \subseteq \mathbb{C}$, the smallest axially symmetric open set containing $D$ is denoted by $\Omega_{D}$ and is given by

$$
\Omega_{D}=\{q=u+I v \mid z=u+i v \in D, I \in \mathbb{S}\} .
$$


We now introduce some terminology following [18].

Definition 2.2. A function $F: D \subseteq \mathbb{C} \rightarrow \mathbb{R}_{n} \otimes \mathbb{C}$ defined by $F(z)=\alpha(z)+i \beta(z)$ where $z=u+i v \in D, \alpha, \beta: D \rightarrow \mathbb{R}_{n}$, and where $\alpha(\bar{z})=\alpha(z)$ and $\beta(\bar{z})=-\beta(z)$ whenever $z, \bar{z} \in D$, is called a stem function. Given a stem function $F$, the function $f=\mathcal{I}(F)$ defined by

$$
f(q)=f(u+I v):=\alpha(u, v)+I \beta(u, v)
$$

for any $q \in \Omega_{D}$ is called the slice function induced by $F$.

Remark 2.3. Note that $\mathcal{I}(F)$ is well defined since $\alpha$ and $\beta$ are respectively even and odd functions in the second variable. The function $\mathcal{I}(F)$ is said to be real (see [18]) if the components $\alpha, \beta$ are real-valued.

Definition 2.4. Let $D$ be an open set in $\mathbb{C}$ and let $F(z)=\alpha(z)+i \beta(z), z=u+i v$, be a stem function. Assume $\alpha, \beta: D \rightarrow \mathbb{R}_{n}$ are $\mathcal{C}^{1}$ functions satisfying the CauchyRiemann system

$$
\left\{\begin{array}{l}
\frac{\partial \alpha}{\partial u}-\frac{\partial \beta}{\partial v}=0 \\
\frac{\partial \alpha}{\partial v}+\frac{\partial \beta}{\partial u}=0
\end{array}\right.
$$

The function $f=\mathcal{I}(F): \Omega_{D} \rightarrow \mathbb{R}_{n}$ is called a slice monogenic function, and the set of slice monogenic functions on $\Omega_{D}$ will be denoted by $\mathcal{S M}\left(\Omega_{D}\right)$.

In the paper [8] the authors give a slightly different definition for a class of functions, which we will call s-monogenic:

Definition 2.5. Let $U$ be an open set $\mathbb{R}^{n+1}$. A real differentiable function $f$ : $U \rightarrow \mathbb{R}_{n}$ is said to be $s$-monogenic if, for every $I \in \mathbb{S}$, its restriction $f_{I}$ of $f$ to the complex plane $\mathbb{C}_{I}$ satisfies

$$
\frac{1}{2}\left(\frac{\partial}{\partial u}+I \frac{\partial}{\partial v}\right) f_{I}(u+v I)=0
$$

The definition of slice monogenic functions immediately implies the following result; see [18]:

Proposition 2.6. Let $f: \Omega_{D} \rightarrow \mathbb{R}_{n}$ be a slice monogenic function. Then for every $I \in \mathbb{S}$ the function $f$ satisfies

$$
\frac{1}{2}\left(\frac{\partial}{\partial u}+I \frac{\partial}{\partial v}\right) f(u+I v)=0
$$

on $U \cap \mathbb{C}_{I}$.

In other words:

Corollary 2.7. Slice monogenic functions are s-monogenic.

Conversely, functions s-monogenic are slice monogenic only over axially symmetric domains such that their intersection with any complex plane $\mathbb{C}_{I}$ is connected. These domains will be called axially symmetric slice domains. This partial inverse of the previous corollary is based on the following result (see [12]), which is a consequence of the validity of the Identity Principle on slice domains; see [ 8 . In the case of slice regular functions over quaternions in the sense of [16], an analogous formula appeared in 2] (and see also [11]). 
Theorem 2.8 (Representation Formula). Let $f$ be an s-monogenic function on an axially symmetric slice domain $\Omega \subseteq \mathbb{R}^{n+1}$. Choose any $J \in \mathbb{S}$. Then the following equality holds for all $x=u+v I \in \Omega$ :

$f(u+v I)=\frac{1}{2}[f(u+v J)+f(u-v J)]+I \frac{1}{2}[J[f(u-v J)-f(u+v J)]]=\alpha(u, v)+I \beta(u, v)$.

Moreover, $\alpha$ and $\beta$ depend only on $u, v \in \mathbb{R}$ such that $u+J v \subseteq \Omega$, but do not depend on $J \in \mathbb{S}$.

Since it is immediate to verify that $\alpha$ and $\beta$ satisfy the properties described in Definition 2.4, we therefore have (see also [18]):

Corollary 2.9. Functions s-monogenic on axially symmetric slice domains are slice monogenic.

Formula (2) holds in a greater generality; in fact it holds for slice functions (not necessarily regular) and it holds on axially symmetric domains which are not necessarily slice domains. Indeed in [18 the authors prove the following result:

Theorem 2.10. Let $f$ be a slice function defined on an axially symmetric open set $\Omega$. Then $f$ satisfies the Representation Formula.

Remark 2.11. Consider the set $\mathcal{S M}(\Omega)$ of slice monogenic functions on an axially symmetric open set $\Omega$. The set is clearly closed under addition, but it is a more interesting fact that it is also possible to define a notion of product between two of its elements: consider two slice monogenic functions $f=\mathcal{I}(F), g=\mathcal{I}(G)$. We define (see [18]) $f \cdot g:=\mathcal{I}(F G)$, where $F G$ is the standard pointwise multiplication of the stem functions:

$$
F G=(\alpha+i \beta)(\gamma+i \delta)=(\alpha \gamma-\beta \delta)+i(\alpha \delta+\beta \gamma) .
$$

It is immediate to verify that $\mathcal{S M}(\Omega)$ is a ring under these two operations. Note that this product coincides with the $*$-product of s-monogenic functions defined in [9] in the case in which $\Omega$ is a slice domain. In fact we have

$$
\mathcal{I}(F G)=\mathcal{I}(F) * \mathcal{I}(G) .
$$

The set $\mathcal{S M}(\Omega)$ can also be considered as a right module on $\mathbb{R}_{n}$.

An s-monogenic function fulfills a property called the Splitting Lemma (see []) which allows us to decompose it as the sum of holomorphic functions. This property also holds for slice monogenic functions:

Lemma 2.12 (Splitting Lemma). Let $\Omega$ be an axially symmetric set and let $f \in$ $\mathcal{S} \mathcal{M}(\Omega)$. For any $I \in \mathbb{S}$ and any choice of $I_{2}, \ldots, I_{n} \in \mathbb{S}$ mutually orthogonal and orthogonal to $I$, there exist $2^{n-1}$ holomorphic functions $F_{A}: U \cap \mathbb{C}_{I} \rightarrow \mathbb{C}_{I}$ such that

$$
f_{I}(x+I y)=\sum_{A} F_{A}(x+I y) I_{A} .
$$


Proof. By hypothesis, $f=\mathcal{I}(F)$, where $F(z)=\alpha(z)+i \beta(z), z=x+i y, \alpha, \beta$ satisfy the Cauchy-Riemann system and $f_{I}(x+I y)=\alpha(x+I y)+I \beta(x+I y)$. Set $I_{1}=I$ and consider $2^{n-1}$ mutually orthogonal elements $I_{2}, \ldots, I_{n} \in \mathbb{S}$, all of which are orthogonal to $I_{1}$, and take them as a basis for $\mathbb{R}_{n}$. Since $\alpha, \beta$ are $\mathbb{R}_{n}$-valued we can write

$$
f_{I}=\sum_{A \in \wp(1, \ldots, n)} a_{A} I_{A}+I \sum_{A \in \wp(1, \ldots, n)} b_{A} I_{A}=\sum_{A \in \wp(2, \ldots, n)}\left(c_{0 A}+c_{1 A} I\right) I_{A} .
$$

Since, with obvious meaning of the symbols, $\left(\partial_{x}+I \partial_{y}\right) f_{I}=0$, we obtain the equations

$$
\begin{aligned}
& \partial_{x} c_{0 A}-\partial_{y} c_{1 A}=0, \\
& \partial_{y} c_{0 A}+\partial_{x} c_{1 A}=0,
\end{aligned}
$$

which are equivalent to the request that the functions $F_{A}=c_{0 A}+I c_{1 A}$ are holomorphic.

\section{Runge theorems}

In this section we will prove several versions of a slice monogenic analog of the classical Runge theorems, for which we refer the reader to [19. To start with, we briefly discuss the notion of a "quotient" of two slice monogenic functions. As is well known (see [1]), in a Clifford algebra $\mathbb{R}_{n}, n \geq 3$, there are zero divisors, thus the norm defined in $\mathbb{R}_{n},|a|^{2}:=\sum_{A} a_{A}^{2}$ cannot be multiplicative; i.e. in general $|a b| \neq|a||b|$. Note also that in general $|a|^{2}$ does not coincide with $a a^{c}$ : in fact $|a|^{2}$ equals the scalar part of $a a^{c}$. Whenever $a a^{c}=\sum_{A} a_{A}^{2}$ and $a \neq 0$ the Clifford number $a$ is invertible and its inverse is $a^{c} / a a^{c}$. Thus it is obvious that in general, given a function, it will not be possible to define its inverse without adding further hypotheses. If a function $F=\alpha+i \beta$ is a real nonidentically zero slice function, then it is invertible and its inverse is

$$
F^{-1}=\frac{1}{\alpha^{2}+\beta^{2}}(\alpha-i \beta)
$$

Given a stem function $F: D \subseteq \mathbb{C} \rightarrow \mathbb{R}_{n}, F(z)=\alpha(z)+i \beta(z)$, let us define the function $F^{c}$ (see [18]) by $F^{c}(z)=\alpha(z)^{c}+i \beta(z)^{c}$. Let $f=\mathcal{I}(F)$ and let $N(f)$ be the function $\mathcal{I}\left(F F^{c}\right)$. Note that

$$
\begin{aligned}
F(z) F^{c}(z) & =(\alpha(z)+i \beta(z))\left(\alpha(z)^{c}+i \beta(z)^{c}\right) \\
& =\alpha(z) \alpha(z)^{c}-\beta(z) \beta(z)^{c}+i\left(\alpha(z) \beta(z)^{c}+\beta(z) \alpha(z)^{c}\right)
\end{aligned}
$$

and

$$
\begin{aligned}
F^{c}(z) F(z) & =\left(\alpha(z)^{c}+i \beta(z)^{c}\right)(\alpha(z)+i \beta(z)) \\
& =\alpha(z)^{c} \alpha(z)-\beta(z)^{c} \beta(z)+i\left(\alpha(z)^{c} \beta(z)+\beta(z)^{c} \alpha(z)\right) .
\end{aligned}
$$

Remark 3.1. The noncommutative setting implies that, in general, $F F^{c} \neq F^{c} F$. Moreover, in general $F F^{c}$ (and similarly $F^{c} F$ ) is not a real slice function (according to Remark 2.3) since $\alpha(z) \alpha(z)^{c}-\beta(z) \beta(z)^{c}$ and $\alpha(z) \beta(z)^{c}+\beta(z) \alpha(z)^{c}$ are not realvalued functions. 
In the sequel, by $Z_{g}$ we will denote the set of zeros of a function $g$. We have the following:

Proposition 3.2. Let $F: D \subseteq \mathbb{C} \rightarrow \mathbb{R}_{n}$ be a stem function such that $F^{c} F$ is a real slice function not identically zero on a dense subset of $D$. Let $\Omega_{D}=\{x=$ $u+I v \mid z=u+i v \in D, I \in \mathbb{S}\}$ and $f=\mathcal{I}(F)$ be a slice monogenic function. Then the slice monogenic inverse of $f$ is the function

$$
f^{-1}:=\mathcal{I}\left(\left(F^{c} F\right)^{-1} F^{c}\right)
$$

defined on $\Omega_{D} \backslash Z_{N\left(F F^{c}\right)}$.

Proof. The stem function $\left(\left(F^{c} F\right)^{-1} F^{c}\right)(z)=\gamma(z)+i \delta(z)$ has components $\gamma, \delta$ satisfying the Cauchy-Riemann system because it is obtained from $F, F^{c}$, which satisfy the Cauchy-Riemann system. Moreover, we have

$$
\left(\left(F^{c} F\right)^{-1} F^{c}\right) F=\left(F^{c} F\right)^{-1} F^{c} F=1 .
$$

Finally, observe that $F F^{c}$ is a stem function with a real component, so

$$
\mathcal{I}\left(\left(F^{c} F\right)^{-1} F^{c}\right)=\mathcal{I}\left(\left(F^{c} F\right)^{-1}\right) \mathcal{I}\left(F^{c}\right)
$$

and the singularities of the function come from those of $\mathcal{I}\left(\left(F^{c} F\right)^{-1}\right)$. The statement follows.

Remark 3.3. The Identity Principle does not hold for slice monogenic functions unless they are defined on domains intersecting the real axis. Thus $F^{c} F$ can be identically zero even though $F$ does not vanish identically; see Remark 12 in [18.

Remark 3.4. In [9, we have introduced the notion of a reciprocal of an s-monogenic function $f$ defined on an axially symmetric slice domain, and we have denoted it by $f^{-*}$. In order to do that, we have to introduce the conjugate $f^{c}$ of the function $f$ and its symmetrization $f^{s}=f * f^{c}$; see [9]. Outside the set $Z_{f^{s}}$ of the zeros of $f^{s}$ we can define the so-called slice monogenic reciprocal of $f$ as $\left(f^{s}\right)^{-1} f^{c}$. By its definition, the s-monogenic reciprocal of $f$ is always defined if $f$ is not identically zero, since for s-monogenic functions defined on slice domains the Identity Principle does hold.

Among the slice monogenic function we now consider, in particular, a polynomial $Q$ in the paravector variable $x$ of the form $a(x)=x^{n} a_{n}+x^{n-1} a_{n-1}+\ldots+x a_{1}+a_{0}$, which is a slice regular function obtained as $a=\mathcal{I}(A)$ from the polynomial

$$
A(z)=z^{n} a_{n}+z^{n-1} a_{n-1}+\ldots+z a_{1}+a_{0}=\sum_{k=0}^{n}(u+i v)^{k} a_{k}=\sum_{k=0}^{n}\left(u_{k}+i v_{k}\right) a_{k}
$$

where

$$
u_{k}=\sum_{j \equiv 0(\bmod 4)}\left(\begin{array}{l}
k \\
j
\end{array}\right) u^{k-j} v^{j}-\sum_{j \equiv 2(\bmod 4)}\left(\begin{array}{l}
k \\
j
\end{array}\right) u^{k-j} v^{j}
$$

and

$$
v_{k}=\sum_{j \equiv 1(\bmod 4)}\left(\begin{array}{l}
k \\
j
\end{array}\right) u^{k-j} v^{j}-\sum_{j \equiv 3(\bmod 4)}\left(\begin{array}{l}
k \\
j
\end{array}\right) u^{k-j} v^{j}
$$


are real quantities. We have

$$
A^{c} A=\left(\sum_{k=0}^{n}\left(u_{k}+i v_{k}\right) a_{k}^{c}\right)\left(\sum_{k=0}^{n}\left(u_{k}+i v_{k}\right) a_{k}\right)=\sum_{k=0}^{2 n}\left(u_{k}+i v_{k}\right) d_{k}
$$

where $d_{k}=\sum_{j=0}^{k} a_{j}^{c} a_{k-j}$.

Remark 3.5. The expressions (3), (4) and (5) clearly show that $A A^{c}$ is a slice function. Its coefficients $d_{k}$ are such that $d_{k}^{c}=\sum_{j=0}^{k}\left(a_{j}^{c} a_{k-j}\right)^{c}=\sum_{j=0}^{k} a_{k-j}^{c} a_{j}=$ $d_{k}$, but they are not necessarily real.

Definition 3.6. Given two polynomials $a=\mathcal{I}(A)$ and $b=\mathcal{I}(B)$ such that $A^{c} A$ is real and not identically zero, we call the (left) rational function a function of the form $a^{-1} b:=\mathcal{I}\left(\left(A^{c} A\right)^{-1} A^{c} B\right)$.

In an analogous way, one can define right rational functions, i.e. functions of the form $a^{-1} b:=\mathcal{I}\left(B A^{c}\left(A^{c} A\right)^{-1}\right)$, and the two theories are equivalent. Rational slice monogenic functions admit the following characterization:

Proposition 3.7. A slice monogenic function $f: \Omega \rightarrow \mathbb{R}_{n}$ is rational if and only if for any $I \in \mathbb{S}$ and any choice of $I_{2}, \ldots, I_{n}$ completion to basis of $\mathbb{R}_{n}$ there are $2^{n-1}$ rational functions $R_{A}$ such that

$$
f_{I}(u+I v)=\sum_{A} R_{A}(u+I v) I_{A},
$$

for any $u+I v \in \Omega \cap \mathbb{C}_{I}$.

Proof. Assume that $f$ is rational. By assumption there exist two polynomial stem functions $A$ and $B$, where $A(z)=\alpha(z)+i \beta(z)$ and $B(z)=\gamma(z)+i \delta(z)$, and $A A^{c}$ is real (in the sense of Remark 2.3) such that $f=a^{-1} b=\mathcal{I}\left(\left(A A^{c}\right)^{-1} A^{c} B\right)$. Then the restriction of $f$ to $\mathbb{C}_{I}$ is given by

$$
\begin{gathered}
f_{I}(u+I v)=\left(\left(A A^{c}\right)^{-1} A^{c} B\right)(u+I v) \\
=\left(\alpha \alpha^{c}-\beta \beta^{c}+I\left(\alpha^{c} \beta+\beta^{c} \alpha\right)\right)^{-1}\left(\alpha^{c} \gamma-\beta^{c} \delta+I\left(\alpha^{c} \delta+\beta^{c} \gamma\right)\right)(u+I v) .
\end{gathered}
$$

Now choose a completion $I_{2}, \ldots, I_{n}$ of $I=I_{1}$ to a basis of $\mathbb{R}_{n}$ such that $I_{i} I_{j}+I_{j} I_{i}=$ $-2 \delta_{i j}$ and note that the polynomial $\alpha^{c} \gamma-\beta^{c} \delta+I\left(\alpha^{c} \delta+\beta^{c} \gamma\right)$ can be written in the form

$$
\sum_{A \in \wp(2, \ldots, n)} P_{A}(u+I v) I_{A} .
$$

Since the function $\left(\alpha \alpha^{c}-\beta \beta^{c}+I\left(\alpha^{c} \beta+\beta^{c} \alpha\right)\right)^{-1}$ has coefficients in $\mathbb{C}_{I}$, it is immediate that (7) is of the form (6). Conversely, let us assume that for a chosen $I \in \mathbb{S}$ the Splitting Lemma applied to the restriction $f_{I}$ of $f$ to $\mathbb{C}_{I}$ can be written in the form (6). The representation formula shows that the function $f$ can be obtained as

$$
\begin{aligned}
f(x) & =\left[\sum_{A} R_{A}(Z) I_{A}+\sum_{A} R_{A}(\bar{Z}) I_{A}\right]+I_{x} I\left[\sum_{A} R_{A}(\bar{Z}) I_{A}-\sum_{A} R_{A}(Z) I_{A}\right] \\
& =\alpha(u, v)+I_{x} \beta(u, v),
\end{aligned}
$$

where $Z=u+I v$ and $\bar{Z}=u-I v$, and $\alpha, \beta$ depend only on $u, v$ but are independent of $I$. We have to show that $f$ is of the form $f=a^{-1} b$. Every rational function 
$R_{A}: \Omega \cap \mathbb{C}_{I} \rightarrow \mathbb{C}_{I}$ can be written as $R_{A}(Z)=P_{A}(Z) / Q_{A}(Z)$, where $P_{A}$ and $Q_{A}$ are polynomials. We also have

$$
\begin{aligned}
\alpha(u, v) & =\sum_{A}\left(\frac{P_{A}(Z)}{Q_{A}(Z)}+\frac{P_{A}(\bar{Z})}{Q_{A}(\bar{Z})}\right) I_{A} \\
& =\sum_{A} \mathcal{Q}_{A}^{-1}(u, v)\left(Q_{A}(\bar{Z}) P_{A}(Z)+Q_{A}(\bar{Z}) P_{A}(\bar{Z})\right) I_{A},
\end{aligned}
$$

where

$$
\begin{aligned}
\mathcal{Q}_{A}(u, v) & =Q_{A}(Z) Q_{A}(\bar{Z})=\left(\sum_{r=0}^{m} Z^{r} a_{r}\right)\left(\sum_{r=0}^{m} \bar{Z}^{r} a_{r}\right) \\
& =\sum_{s=0}^{2 m}\left(Z^{r} \bar{Z}^{s-r}+Z^{s-r} \bar{Z}^{r}\right) a_{r} a_{s-r}
\end{aligned}
$$

and $\mathcal{Q}_{A}$ is defined on $\Omega \cap \mathbb{C}_{I}$. Now note that $\left(Z^{r} \bar{Z}^{s-r}+Z^{s-r} \bar{Z}^{r}\right)$ is real. Thus it depends only on $u, v$ but not on $I \in \mathbb{S}$, while $a_{r} \in \mathbb{C}_{I}$. Setting

$$
\mathcal{Q}(u, v):=\prod_{A} \mathcal{Q}_{A}(u, v)
$$

we can write

$$
\begin{aligned}
\alpha(u, v) & =\mathcal{Q}(u, v)^{-1}\left[\sum \prod_{B \neq A} \mathcal{Q}_{B}(u, v)\left(Q_{A}(\bar{Z}) P_{A}(Z)+Q_{A}(\bar{Z}) P_{A}(\bar{Z})\right) I_{A}\right] \\
& =\mathcal{Q}(u, v)^{-1} \mathcal{A}(u, v),
\end{aligned}
$$

and, in an analogous way, we obtain

$$
\begin{aligned}
\beta(u, v) & =\mathcal{Q}(u, v)^{-1} I\left[\sum \prod_{B \neq A} \mathcal{Q}_{B}(u, v)\left(Q_{A}(\bar{Z}) P_{A}(Z)+Q_{A}(\bar{Z}) P_{A}(\bar{Z})\right) I_{A}\right] \\
& =\mathcal{Q}(u, v)^{-1} \mathcal{B}(u, v),
\end{aligned}
$$

where $\mathcal{Q}$ has coefficients in $\mathbb{C}_{I}$ while the polynomials $\mathcal{A}, \mathcal{B}$ have coefficients in $\mathbb{R}_{n}$. Finally, we obtain that in the open set $\Omega$ the function $f$ can be written as

$$
f(x)=\mathcal{Q}^{-1}\left(\mathcal{A}+I_{x} \mathcal{B}\right)\left(u+I_{x} v\right)=\left(\left(\mathcal{Q}^{c} \mathcal{Q}\right)^{-1} \mathcal{Q}^{c}\right)\left(\mathcal{A}+I_{x} \mathcal{B}\right)\left(u+I_{x} v\right),
$$

and so

$$
f=\mathcal{I}\left(\left(\mathcal{Q}^{c} \mathcal{Q}\right)^{-1} \mathcal{Q}^{c}(\mathcal{A}+i \mathcal{B})\right),
$$

where $\mathcal{Q}^{c} \mathcal{Q}$ is a real function nonidentically zero since the coefficients of $\mathcal{Q}$ belong to the subalgebra $\mathbb{C}_{I}$ of $\mathbb{R}_{n}$.

By following the previous discussion it is easy to show the following result:

Proposition 3.8. The singularities of a rational function are isolated $(n-1)$ spheres of the form $u+\mathbb{S} v$.

Proof. Let $r=a^{-1} b$ be a rational function. Its singularities come from the set of zeros of the function $A^{c} A$ which are isolated points, so the singularities are isolated $(n-1)$-spheres (in particular, a real point when the sphere has radius equal to zero). 
For a study of the poles of a slice regular function defined over a ball, we refer the reader to 20 .

Remark 3.9. The proof of the previous proposition shows that if $x_{0}$ is a pole of a rational function, then all the points of the sphere defined by $x_{0}$ are poles. We point out that the poles belonging to a sphere have order less than or equal to $\operatorname{deg} a$. There cannot be essential singularities.

Definition 3.10. We will say that a singularity $x=x_{0}$ of a function $f$ is a pole if it belongs to an isolated $(n-1)$-sphere of singularities and $\lim _{x \rightarrow x_{0}}|f(x)|=+\infty$.

Proposition 3.8 has the following immediate consequence:

Corollary 3.11. The singularities of a rational function are all poles.

We are now ready to prove the analogue of the Runge theorem. By the symbol $\overline{\mathbb{R}^{n+1}}$ we will denote $\mathbb{R}^{n+1} \cup\{\infty\}$.

Theorem 3.12. Let $K$ be an axially symmetric compact set in $\mathbb{R}^{n+1}$, and let $A$ be a set having a point in each connected component of $\overline{\mathbb{R}^{n+1}} \backslash K$. For any axially symmetric open set $\Omega \supset K$, for every $f \in \mathcal{S M}(\Omega)$ and for any $\varepsilon>0$ there exists a rational function $r$ whose poles are spheres in $A$ such that

$$
|f(x)-r(x)|<\varepsilon
$$

for all $x \in K$.

Proof. Let us consider the restriction of the function $f$ to a complex plane $\mathbb{C}_{I}$. The proof of the Splitting Lemma 2.12 shows that for every $I \in \mathbb{S}$ and every choice of a completion of $I$ to a basis of $\mathbb{R}_{n}$ of unit vectors mutually orthogonal, there are $2^{n-1}$ holomorphic functions $F_{A}: \Omega \cap \mathbb{C}_{I} \rightarrow \mathbb{C}_{I}$ such that for any $z=u+I v$, the restriction of $f$ to $\mathbb{C}_{I}$ can be written as

$$
f_{I}(z)=\sum_{A} F_{A}(z) I_{A}
$$

By the standard Runge theorem (see [19]) we can find $2^{n-1}$ rational functions $R_{A}(u+I v)$ with poles in $A \cap \mathbb{C}_{I}$ such that

$$
\left|F_{A}(u+I v)-R_{A}(u+I v)\right|<\frac{\varepsilon}{2^{n}} \quad \forall u+I v \in \Omega \cap \mathbb{C}_{I} .
$$

Since $\Omega \cap \mathbb{C}_{I}$ is symmetric with respect to the real axis, the structure formula of Theorem 2.8 allows us to extend the function $r(u+I v)$ to the whole $\Omega$ as

$$
r\left(u+I_{q} v\right)=\frac{1}{2}\left[r(u+I v)+r(u-I v)+I_{q} I[r(u-I v)-r(u+I v)]\right] .
$$

Let us now consider $|f(q)-r(q)|$. Using again the structure formula, we have

$$
\begin{gathered}
|f(q)-r(q)|=\mid \frac{1}{2}\left[f(u+I v)+f(u-I v)+I_{q} I[f(u-I v)-f(u+I v)]\right] \\
-\frac{1}{2}\left[r(u+I v)+r(u-I v)+I_{q} I[r(u-I v)-r(u+I v)]\right] \mid .
\end{gathered}
$$


By the Splitting Lemma we can write

$$
\begin{gathered}
=\mid \frac{1}{2}\left[\sum_{A} F_{A}(u+I v) I_{A}+F_{A}(u-I v) I_{A}\right. \\
\left.\left.\quad+I_{q} I\left[\sum_{A} F_{A}(u-I v) I_{A}-\sum_{A} F_{A}(u+I v) I_{A}\right)\right]\right] \\
-\frac{1}{2}\left[\sum_{A} R_{A}(u+I v) I_{A}+\sum_{A} R_{A}(u-I v) I_{A}\right. \\
\left.+I_{q} I\left[\sum_{A} R_{A}(u-I v) I_{A}-\sum_{A} R_{A}(u+I v) I_{A}\right]\right] \mid \\
=\frac{1}{2}\left[\sum_{A}\left|F_{A}(u+I v)-R_{A}(u+I v)\right|\right. \\
\quad+\sum_{A}\left|F_{A}(u-I v)-R_{A}(u-I v)\right| \\
\quad+\sum_{A}\left|F_{A}(u-I v)-R_{A}(u-I v)\right| \\
\left.-\sum_{A}\left|F_{A}(u+I v)-R_{A}(u+I v)\right|\right] .
\end{gathered}
$$

Thus, using (8) we obtain

$$
|f(q)-r(q)| \leq \varepsilon
$$

The result follows.

In particular, we have the following result which allows us to approximate a slice regular function with polynomials:

Theorem 3.13. Let $K$ be an axially symmetric compact set such that $\overline{\mathbb{R}^{n+1}} \backslash K$ is connected (and $\overline{\mathbb{C}}_{I} \backslash\left(K \cap \mathbb{C}_{I}\right)$ is connected for all $I \in \mathbb{S}$ ) and let $f \in \mathcal{S R}(\Omega)$ where $\Omega \supset K$ is an open set. There exists a sequence $\left\{P_{n}\right\}$ of polynomials such that $P_{n}(q) \rightarrow f(q)$ uniformly on $K$.

Proof. Our assumptions imply that $\overline{\mathbb{R}^{n+1}} \backslash K$ has only one component. Thus we can apply Theorem 3.12 with $A=\{\infty\}$.

We also have the following version of the Runge theorem which holds for open sets:

Theorem 3.14. Let $\Omega$ be an axially symmetric open set in $\overline{\mathbb{R}^{n+1}}$, let $A$ be a set having a point in each connected component of $\overline{\mathbb{R}^{n+1}} \backslash \Omega$ and let $f \in \mathcal{S R}(\Omega)$. Then $f$ can be approximated by a sequence of rational functions $\left\{r_{n}\right\}$ having their poles in $A$ uniformly on every compact set in $\Omega$. If $\overline{\mathbb{R}^{n+1}} \backslash \Omega$ is a connected set, then we can set $A=\{\infty\}$ and $f$ can be approximated by polynomials uniformly on every compact set in $\Omega$.

Proof. Consider a sequence $\left\{K_{n}\right\}$ of axially symmetric compact subsets of $\Omega$ such that $K_{n} \Subset K_{n+1}$, any compact set in $\Omega$ is contained in $K_{n}$ for some $n$, and each component of $\overline{\mathbb{R}^{n+1}} \backslash K_{n}$ contains a component of $\overline{\mathbb{R}^{n+1}} \backslash \Omega$ for all $n$. This last assumption implies that each component of $\overline{\mathbb{R}^{n+1}} \backslash K_{n}$ contains a point in $A$. By 
Theorem 3.12 there exists a function $r_{n}$ having poles in $A$ such that

$$
\left|f(q)-r_{n}(q)\right|<\frac{1}{n}, \quad q \in K_{n} .
$$

If $K$ is any axially symmetric compact subset of $\Omega$, then our assumptions assure that there exists $N$ such that $K \subset K_{n}$ for all $n \geq N$. Thus

$$
\left|f(q)-r_{n}(q)\right|<\frac{1}{n}, \quad q \in K, n \geq N
$$

and this completes the proof.

By suitably modifying the proof of the previous result one can prove the following theorem:

Theorem 3.15. Let $\Omega_{1}, \Omega_{2}$ be axially symmetric open sets in $\mathbb{R}^{n+1}$ such that $\Omega_{1} \subset \Omega_{2}$ and each connected component of $\overline{\mathbb{R}^{n+1}} \backslash \Omega_{1}$ intersects $\overline{\mathbb{R}^{n+1}} \backslash \Omega_{2}$. Then every function in $\mathcal{S R}\left(\Omega_{1}\right)$ can be approximated by functions in $\mathcal{S} \mathcal{R}\left(\Omega_{2}\right)$ uniformly on every compact set in $\Omega_{1}$.

Definition 3.16. A pair of axially symmetric open sets $\Omega_{1}, \Omega_{2}$ in $\mathbb{R}^{n+1}$ such that $\Omega_{1} \subset \Omega_{2}$ and each connected component of $\overline{\mathbb{R}^{n+1}} \backslash \Omega_{1}$ intersects $\overline{\mathbb{R}^{n+1}} \backslash \Omega_{2}$ is said to be a Runge pair.

We also have the following slice monogenic version of the Mittag-Leffler theorem:

Proposition 3.17. Let $\Omega_{1}, \Omega_{2}$ be axially symmetric open sets in $\mathbb{R}^{n+1}$ and let $f \in \mathcal{S R}\left(\Omega_{1} \cap \Omega_{2}\right)$. Then there exist $f_{j} \in \mathcal{S R}\left(\Omega_{j}\right), j=1,2$, such that

$$
f(q)=f_{1}(q)-f_{2}(q), \quad \text { on } \Omega_{1} \cap \Omega_{2} .
$$

Proof. If we assume that $\Omega_{1}, \Omega_{2}$ are bounded with piecewise smooth boundary and that $f$ is slice regular on the closure of $\Omega_{1} \cap \Omega_{2}$, then the statement follows from the Cauchy formula. In fact, set $\Gamma_{1}:=\partial\left(\Omega_{1}\right) \cap \bar{\Omega}_{2}$ and $\Gamma_{2}:=\partial\left(\Omega_{2}\right) \cap \bar{\Omega}_{1}$, and recall that the Cauchy kernel is

$$
S^{-1}(s, q)=-\left(q^{2}-2 \operatorname{Re}(s) q+|s|^{2}\right)^{-1}(q-\bar{s}) .
$$

Then we have

$f(q)=\int_{\partial\left(\Omega_{1} \cap \Omega_{2}\right) \cap \mathbb{C}_{I}} S^{-1}(s, q) d s_{I}=\int_{\Gamma_{1} \cap \mathbb{C}_{I}} S^{-1}(s, q) d s_{I}+\int_{-\left(\Gamma_{2} \cap \mathbb{C}_{I}\right)} S^{-1}(s, q) d s_{I} f(s)$, where the minus sign refers to the orientation of two curves $\Gamma_{2} \cap \mathbb{C}_{I}$ in the plane $\mathbb{C}_{I}$. If we set

$$
f_{1}(q)=\int_{\left(\Gamma_{1} \cap \mathbb{C}_{I}\right)^{+}} S^{-1}(s, q) d s_{I}, \quad f_{2}(q)=\int_{-\left(\Gamma_{2} \cap \mathbb{C}_{I}\right)^{+}} S^{-1}(s, q) d s_{I} f(s),
$$

we get $f_{j} \in \mathcal{S R}\left(\Omega_{j}\right), j=1,2$, and the statement follows. In the general case, consider a sequence $\Omega_{j k}$ of open axially symmetric subsets of $\Omega_{j k}, j=1,2$, such that $\bigcup_{k} \Omega_{j k}=\Omega_{j}, \Omega_{j k} \Subset \Omega_{j, k+1}$. Assume that $\partial \Omega_{j k}$ is piecewise smooth and that $\left(\Omega_{1} \cup \Omega_{2}, \Omega_{1 k} \cup \Omega_{2 k}\right)$ form a Runge pair for every $k$. Consider the restriction of $f$ to the open set $\Omega_{1 k} \cap \Omega_{2 k}$. By the preceding discussion we have that there exist $f_{j k} \in \mathcal{S R}\left(\Omega_{j k}\right)$ such that

$$
f_{\mid \Omega_{1 k} \cap \Omega_{2 k}}=f_{1 k}-f_{2 k}
$$

on $\Omega_{1 k} \cap \Omega_{2 k}$. Repeating the procedure for the index $k+1$ and taking the difference, we get

$$
f_{1, k+1}-f_{1 k}=f_{2, k+1}-f_{2 k}
$$


on $\Omega_{1 k} \cap \Omega_{2 k}$. This difference defines a slice regular function $F_{k}$ on $\Omega_{1 k} \cup \Omega_{2 k}$. By Theorem 3.15, there exist $G_{k} \in \mathcal{S R}\left(\Omega_{1} \cup \Omega_{2}\right)$ such that

$$
\left|F_{k}(q)-G_{k}(q)\right| \leq \frac{1}{2^{k}}, \quad \Omega_{1, k-1} \cup \Omega_{2, k-1} .
$$

Now define

$$
f_{j}(q):=f_{j 1}(q)+\sum_{k=1}^{+\infty}\left(F_{k}(q)-G_{k}(q)\right), \quad j=1,2 .
$$

Since we can write

$$
\begin{gathered}
f_{j}(q)=f_{j 1}(q)+\sum_{k=1}^{+\infty}\left(f_{1, k+1}(q)-f_{1 k}(q)-G_{k}(q)\right) \\
=f_{j 1}(q)+\sum_{k=1}^{+\infty}\left(f_{j, k+1}(q)-f_{j k}(q)-G_{k}(q)\right) \\
=f_{j N}(q)+\sum_{k=N}^{+\infty}\left(F_{k}(q)-G_{k}(q)\right)-\sum_{k=1}^{N-1} G_{k}(q),
\end{gathered}
$$

we have that $f_{j} \in \mathcal{S R}\left(\Omega_{j}\right)$ and the statement follows.

Proposition 3.18. If an open set $U$ in $\mathbb{R}^{n+1}$ is axially symmetric, then it is a domain of slice monogenicity.

Proof. Assume that $U$ is an axially symmetric open set and consider its intersection with a complex plane $\mathbb{C}_{I}$ given an open set which is a domain of holomorphy on that plane. This fact implies that there is a function $f$ which cannot be holomorphically extended outside $U \cap \mathbb{C}_{I}$. Call $F$ the slice monogenic function obtain by $f$ through monogenic extension. If $F$ was extendable as a slice monogenic function, then also its restriction would be holomorphic over a set $U^{\prime} \cap \mathbb{C}_{I} \supset U \cap \mathbb{C}_{I}$.

\section{Slice REgular FUnCtions}

The results that we have stated for slice monogenic functions can be repeated for a different class of functions: the so-called slice regular functions, originally introduced in [16] and [17. These functions are defined on open sets of the space of real quaternions $\mathbb{H}$ and are $\mathbb{H}$-valued. Since the algebra of quaternions is a division algebra, some of the results in the previous sections can be proved under weaker assumptions, and, for this reason, we will repeat them in this section. Let us start by listing some basic notions on slice regular functions. A real quaternion $q$ will be written as $q=x_{0}+x_{1} \mathbf{i}+x_{2} \mathbf{j}+x_{3} \mathbf{k}$. The purely imaginary quaternions are elements of the form $x_{1} \mathbf{i}+x_{2} \mathbf{j}+x_{3} \mathbf{k}$; those of norm 1 form a 2 -sphere in $\mathbb{H}$ denoted by $\mathbb{S}$. As in Section 2, we will introduce the terminology for the open sets in $\mathbb{H}$. There are some that are important in our theory:

Definition 4.1. Let $\Omega \subseteq \mathbb{H}$. We say that $\Omega$ is axially symmetric if, for all $x+I y \in$ $\Omega$, the 2 -sphere $x+\mathbb{S} y=\{x+J y \mid J \in \mathbb{S}\}$ is contained in $\Omega$. A connected open set intersecting the real axis and such that its intersection with any complex plane $\mathbb{C}_{I}$ is connected is called a slice domain.

Given an open set $D \subseteq \mathbb{C}$, the least axially symmetric open set containing $D$ is again denoted by $\Omega_{D}$, and it is defined as in Section 2 . 
The definition of slice regular functions that we will use is slightly different from the one in [16] and is given as follows; see [18].

Definition 4.2. A function $F: D \subseteq \mathbb{C} \rightarrow \mathbb{H}_{\mathbb{C}}$ defined by $F(z)=\alpha(z)+i \beta(z)$ where $z=u+i v \in D, \alpha, \beta: D \rightarrow \mathbb{H}$ satisfying $\alpha(\bar{z})=\alpha(z)$ and $\beta(\bar{z})=-\beta(z)$ whenever $z, \bar{z} \in D$ is a stem function. Given a stem function $F$, the function $f=\mathcal{I}(F)$, defined by

$$
f(q)=f(u+I v):=\alpha(u, v)+I \beta(u, v)
$$

for any $q \in \Omega_{D}$, is called is said to be the slice function induced by $F$. A slice function is said to be real if both $\alpha$ and $\beta$ are real-valued.

If $\alpha, \beta: D \rightarrow \mathbb{H}$ are $\mathcal{C}^{1}$ and satisfy the Cauchy-Riemann system,

$$
\left\{\begin{array}{l}
\frac{\partial \alpha}{\partial u}-\frac{\partial \beta}{\partial v}=0 \\
\frac{\partial \alpha}{\partial v}+\frac{\partial \beta}{\partial u}=0
\end{array}\right.
$$

the function $f=\mathcal{I}(F)$ is called a slice regular function.

Slice regular functions satisfy the Splitting Lemma (as the slice regular functions in the sense of [16 do; see for example [17]). Since the proof is analogous to the one given for slice monogenic functions, we omit it and just give the statement:

Lemma 4.3 (Splitting Lemma). Let $\Omega$ be an axially symmetric set and let $f \in$ $\mathcal{S R}(\Omega)$. For any $I \in \mathbb{S}$ and any choice of $J \in \mathbb{S}$ orthogonal to $I$ there exists two holomorphic functions $F, G: U \cap \mathbb{C}_{I} \rightarrow \mathbb{C}_{I}$ such that

$$
f_{I}(x+I y)=F(x+I y)+G(x+I y) J .
$$

Consider the set $\mathcal{S R}(\Omega)$ on an axially symmetric open set $\Omega$. Besides the notion of sum, defined pointwise, the set can be endowed with the operation of product. If $f=\mathcal{I}(F)$ and $g=\mathcal{I}(G)$ are two slice regular functions we define (see [18]), $f \cdot g:=\mathcal{I}(F G)$, where $F G$ is the standard pointwise multiplication of functions. Once again we can show that $\mathcal{S R}(U)$ is a ring, which can also be considered as a right vector space on $\mathbb{H}$.

4.1. Runge theorems for slice regular functions. As we have done in the case of slice monogenic functions, given a stem function $F: D \subseteq \mathbb{C} \rightarrow \mathbb{H}, F(z)=$ $\alpha(z)+i \beta(z)$, we can define the function $F^{c}$ by $F^{c}(z)=\alpha(z)^{c}+i \beta(z)^{c}$, where $a^{c}$ denotes the quaternionic conjugation $q^{c}=x_{0}-i x_{1}-j x_{2}-k x_{3}$. Let $f=\mathcal{I}(F)$ and denote by $N(f)$ the function $\mathcal{I}\left(F F^{c}\right)$. Note that

$$
\begin{aligned}
F(z) F^{c}(z) & =(\alpha(z)+i \beta(z))\left(\alpha(z)^{c}+i \beta(z)^{c}\right) \\
& =\alpha(z) \alpha(z)^{c}-\beta(z) \beta(z)^{c}+i\left(\alpha(z) \beta(z)^{c}+\beta(z) \alpha(z)^{c}\right) \\
& =|\alpha(z)|^{2}-|\beta(z)|^{2}+i\left(\alpha(z) \beta(z)^{c}+\left(\alpha(z) \beta(z)^{c}\right)^{c}\right)
\end{aligned}
$$

and

$$
\begin{aligned}
F^{c}(z) F(z) & =\left(\alpha(z)^{c}+i \beta(z)^{c}\right)(\alpha(z)+i \beta(z)) \\
& =\alpha(z)^{c} \alpha(z)-\beta(z)^{c} \beta(z)+i\left(\alpha(z)^{c} \beta(z)+\beta(z)^{c} \alpha(z)\right) \\
& =|\alpha(z)|^{2}-|\beta(z)|^{2}+i\left(\alpha(z)^{c} \beta(z)+\left(\alpha(z)^{c} \beta(z)\right)^{c}\right),
\end{aligned}
$$


so, in general, $F F^{c} \neq F^{c} F$. However, here we observe a first difference with the slice monogenic case:

Proposition 4.4. The functions $F F^{c}$ and $F^{c} F$ are real slice functions.

Proof. It is an immediate consequence of the fact that $|\alpha(z)|^{2}-|\beta(z)|^{2}$ is trivially real-valued and $\left(\alpha(z)^{c} \beta(z)+\left(\alpha(z)^{c} \beta(z)\right)^{c}\right)^{c}=\left(\alpha(z)^{c} \beta(z)\right)^{c}+\alpha(z)^{c} \beta(z)$. Thus $\alpha(z)^{c} \beta(z)+\left(\alpha(z)^{c} \beta(z)\right)^{c}$ is real-valued.

By $Z_{g}$ we will denote the set of zeros of a function $g$. By virtue of Proposition 4.4, the notion of a slice regular inverse of a function can be given for all the functions for which $F F^{c}$ is not identically zero:

Proposition 4.5. Let $F: D \subseteq \mathbb{C} \rightarrow \mathbb{H}$ be a stem function such that $F^{c} F$ is not identically zero on a dense subset of $D$. Let $f=\mathcal{I}(F)$ and $\Omega_{D}=\{q=u+I v \mid z=$ $u+i v \in D, I \in \mathbb{S}\}$. Then the slice regular inverse of $f$ is the function

$$
f^{-1}:=\mathcal{I}\left(\left(F^{c} F\right)^{-1} F^{c}\right)
$$

defined on $\Omega_{D} \backslash Z_{N\left(F F^{c}\right)}$.

Proof. See the proof of Proposition 4.4 .

In particular, we now consider a polynomial $Q$ in the variable $q$ of the form $a(q)=q^{n} a_{n}+q^{n-1} a_{n-1}+\ldots+q a_{1}+a_{0}$ which is a slice regular function obtained as $a=\mathcal{I}(A)$ from the polynomial

$$
A(z)=z^{n} a_{n}+z^{n-1} a_{n-1}+\ldots+z a_{1}+a_{0}=\sum_{k=0}^{n}(x+i y)^{k} a_{k}=\sum_{k=0}^{n}\left(u_{k}+i v_{k}\right) a_{k},
$$

where the real quantities $u_{k}, v_{k}$ can be obtained as in (3) and (4).

We have

$$
A^{c} A=\left(\sum_{k=0}^{n}\left(u_{k}+i v_{k}\right) a_{k}^{c}\right)\left(\sum_{k=0}^{n}\left(u_{k}+i v_{k}\right) a_{k}\right)=\sum_{k=0}^{2 n}\left(u_{k}+i v_{k}\right) d_{k},
$$

where $d_{k}=\sum_{j=0}^{k} a_{j}^{c} a_{k-j}$ is a real number. Moreover, $A^{c} A$ is not identically zero if $A$ is not identically zero.

Definition 4.6. Given two polynomials $a=\mathcal{I}(A)$ and $b=\mathcal{I}(B)$, we call the (left) rational function a function of the form $a^{-1} b:=\mathcal{I}\left(\left(A^{c} A\right)^{-1} A B\right)$.

By the same arguments used earlier, it is easy to prove the following result:

Proposition 4.7. The singularities of a rational function are isolated 2-spheres of the form $u_{0}+\mathbb{S} v_{0}$.

Definition 4.8. We will say that a singularity $q=q_{0}$ of a function $f$ is a pole if it belongs to an isolated 2-sphere of singularities and it is such that $\lim _{q \rightarrow q_{0}}|f(q)|=$ $+\infty$.

Proposition 4.7 has the following immediate consequence:

Corollary 4.9. The singularities of a rational function are poles. 
We are now ready to prove the analogue of the Runge theorem:

Theorem 4.10. Let $K$ be an axially symmetric compact set in $\mathbb{H}$, and let $A$ be a set having a point in each connected component of $\overline{\mathbb{H}} \backslash K$. For any axially symmetric open set $\Omega \supset K$, for every $f \in \mathcal{S R}(\Omega)$ and for any $\varepsilon>0$ there exists a rational function $r$ whose poles are spheres in $A$ such that

$$
|f(q)-r(q)|<\varepsilon
$$

for all $q \in K$.

Proof. Let us consider the restriction of the function $f$ to a complex plane $\mathbb{C}_{I}$. The proof of the Splitting Lemma 4.3 shows that for every $I \in \mathbb{S}$ and every $J$ in $\mathbb{S}$ perpendicular to $I$, there are two holomorphic functions $F, G: U \cap \mathbb{C}_{I} \rightarrow \mathbb{C}_{I}$ such that for any $z=u+I v$, the restriction of $f$ to $\mathbb{C}_{I}$ can be written as

$$
f_{I}(z)=F(z)+G(z) J .
$$

By the standard Runge theorem (see [19]) we can find two rational functions $R(u+I v)$ and $S(u+I v)$ with poles in $A \cap \mathbb{C}_{I}$ such that

$$
|F(u+I v)-R(u+I v)|<\frac{\varepsilon}{4} \quad \text { and } \quad|G(u+I v)-S(u+I v)|<\frac{\varepsilon}{4} \quad \forall u+I v \in \Omega \cap \mathbb{C}_{I} .
$$

Since $\Omega \cap \mathbb{C}_{I}$ is symmetric with respect to the real axis, the structure formula allows us to extend the function $r(u+I v)=R(u+I v)+S(u+I v) J$ to the whole $\Omega$ as

$$
r\left(u+I_{q} v\right)=\frac{1}{2}\left[r(u+I v)+r(u-I v)+I_{q} I[r(u-I v)-r(u+I v)]\right],
$$

and it allows us to conclude as in the proof of Theorem 4.10.

In particular, we have the following result which allows us to approximate a slice regular function with polynomials:

Theorem 4.11. Let $K$ be an axially symmetric compact set such that $\overline{\mathbb{H}} \backslash K$ is connected (and $\overline{\mathbb{C}}_{I} \backslash\left(K \cap \mathbb{C}_{I}\right)$ is connected for all $I \in \mathbb{S}$ ) and let $f \in \mathcal{S R}(\Omega)$, where $\Omega \supset K$ is an open set. There exists a sequence $\left\{P_{n}\right\}$ of polynomials such that $P_{n}(q) \rightarrow f(q)$ uniformly on $K$.

Proof. Our assumptions imply that $\overline{\mathbb{H}} \backslash K$ has only one component. Thus we can apply Theorem 4.10 with $A=\{\infty\}$.

We also have the following version of the Runge theorem which holds for open sets:

Theorem 4.12. Let $\Omega$ be an axially symmetric open set in $\overline{\mathbb{H}}$, let $A$ be a set having a point in each connected component of $\overline{\mathbb{H}} \backslash \Omega$ and let $f \in \mathcal{S R}(\Omega)$. Then $f$ can be approximated by a sequence of rational functions $\left\{r_{n}\right\}$ having their poles in $A$ uniformly on every compact set in $\Omega$. If $\overline{\mathbb{H}} \backslash \Omega$ is a connected set, then we can set $A=\{\infty\}$ and $f$ can be approximated by polynomials uniformly on every compact set in $\Omega$. 
By suitably modifying the proof of the previous result one can prove the following results:

Theorem 4.13. Let $\Omega_{1}, \Omega_{2}$ be axially symmetric open sets in $\mathbb{H}$ such that $\Omega_{1} \subset \Omega_{2}$ and each connected component of $\overline{\mathbb{H}} \backslash \Omega_{1}$ intersects $\overline{\mathbb{H}} \backslash \Omega_{2}$. Then every function in $\mathcal{S R}\left(\Omega_{1}\right)$ can be approximated by functions in $\mathcal{S R}\left(\Omega_{2}\right)$ uniformly on every compact set in $\Omega_{1}$.

Proposition 4.14. An axially symmetric open set is a domain of slice regularity.

If one considers the case of slice regular functions in the sense of [16] which are defined on axially symmetric slice domains, an analogous result is proven in [11].

\section{REFERENCES}

[1] F. Brackx, R. Delanghe, F. Sommen, Clifford Analysis, Pitman Res. Notes in Math., 76, 1982. MR697564 (85j:30103)

[2] F. Colombo, G. Gentili, I. Sabadini, A Cauchy kernel for slice regular functions, Ann. Global Anal. Geom., 37 (2010), 361-378. MR2601496

[3] F. Colombo, G. Gentili, I. Sabadini, D.C. Struppa, Non commutative functional calculus: Bounded operators, Complex Analysis and Operator Theory, 4 (2010), 821-843.

[4] F. Colombo, G. Gentili, I. Sabadini, D.C. Struppa, Non commutative functional calculus: Unbounded operators, J. Geom. Phys., 60 (2010), 251-259. MR2587392

[5] F. Colombo, I. Sabadini, On some properties of the quaternionic functional calculus, J. Geom. Anal., 19 (2009), 601-627. MR2496568 (2010h:47024)

[6] F. Colombo, I. Sabadini, F. Sommen, D.C. Struppa, Analysis of Dirac systems and computational algebra, Progress in Mathematical Physics, Vol. 39, Birkhäuser, Boston, 2004. MR2089988 (2005m:30052)

[7] F. Colombo, I. Sabadini, D.C. Struppa, A new functional calculus for noncommuting operators, J. Funct. Anal., 254 (2008), 2255-2274. MR2402108 (2009e:47017)

[8] F. Colombo, I. Sabadini, D.C. Struppa, Slice monogenic functions, Israel J. Math., 171 (2009), 385-403. MR.2520116 (2010e:30039)

[9] F. Colombo, I. Sabadini, D.C. Struppa, Extension properties for slice monogenic functions, Israel J. Math., 177 (2010), 369-389. MR2520116 (2010e:30039)

[10] F. Colombo, I. Sabadini, D.C. Struppa, Duality theorems for slice hyperholomorphic functions, J. Reine Angew. Math., 645 (2010), 85-104. MR2673423

[11] F.Colombo, G. Gentili, I. Sabadini, D.C. Struppa, Extension results for slice regular functions of a quaternionic variable, Adv. Math., 222 (2009), 1793-1808. MR2555912 (2010j:30102)

[12] F. Colombo, I. Sabadini, A structure formula for slice monogenic functions and some of its consequences, Hypercomplex Analysis, Trends in Mathematics, Birkhäuser, 2009, 101-114.

[13] F. Colombo, I. Sabadini, D. C. Struppa, The Pompeiu formula for slice hyperholomorphic functions, to appear in Michigan J. Math. (2011).

[14] C. G. Cullen, An integral theorem for analytic intrinsic functions on quaternions, Duke Math. J., 32 (1965), 139-148. MR0173012 (30:3227)

[15] R. Fueter, Die Funktionentheorie der Differentialgleichungen $\triangle u=0$ und $\triangle \triangle u=0$ mit vier reellen Variablen, Comm. Math. Helv., 7 (1934), 307-330. MR.1509515

[16] G. Gentili, D.C. Struppa, A new approach to Cullen-regular functions of a quaternionic variable, C.R. Acad. Sci. Paris, 342 (2006), 741-744. MR.2227751 (2006m:30095)

[17] G. Gentili, D.C. Struppa, A new theory of regular functions of a quaternionic variable, Adv. Math., 216 (2007), 279-301. MR2353257 (2008h:30052)

[18] R. Ghiloni, A. Perotti, Slice regular functions on real alternative algebras, Adv. Math., 226 (2011), 1662-1691.

[19] W. Rudin, Real and complex analysis, McGraw-Hill Book Co., New York, 1987. MR924157 (88k:00002)

[20] C. Stoppato, Poles of regular quaternionic functions, Compl. Var. and Elliptic Equa., 54 (2009), 1001-1018. MR2572530 (2010k:30062) 
Dipartimento di Matematica, Politecnico di Milano, Via Bonardi, 9, 20133 Milano, ITALY

E-mail address: fabrizio.colombo@polimi.it

Dipartimento di Matematica, Politecnico di Milano, Via Bonardi, 9, 20133 Milano, ITALY

E-mail address: irene.sabadini@polimi.it

Department of Mathematics, Schmid College of Science, Chapman University, Orange, California 92866

E-mail address: struppa@chapman.edu 